\title{
Electron emission from silicon and germanium after swift heavy ion impact
}

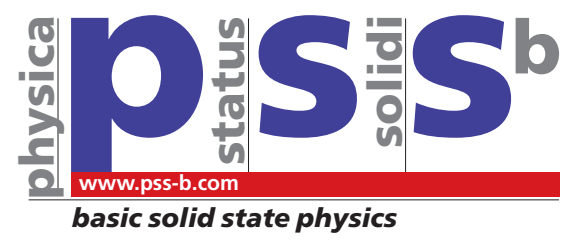

\author{
R. A. Rymzhanov ${ }^{*, 1}$, N. A. Medvedev ${ }^{2}$, and A. E. Volkov ${ }^{1,3,4}$ \\ ${ }^{1}$ FLNR, JINR, Joliot-Curie 6, 141980 Dubna, Russia \\ ${ }^{2}$ Center for Free-Electron Laser Science at DESY, Notkestr. 85, 22607 Hamburg, Germany \\ ${ }^{3}$ NRC Kurchatov Institute, Kurchatov Sq. 1, 123182 Moscow, Russia \\ ${ }^{4}$ LPI of the Russian Academy of Sciences, Leninskij Prospekt, 53, 119991 Moscow, Russia
}

Received 31 May 2014, accepted 21 August 2014

Published online 24 November 2014

Keywords complex dielectric function, electron emission, inelastic mean free path, Monte Carlo simulations, swift heavy ion track

* Corresponding author: e-mail r.a.rymzhanov@gmail.com, Phone: +7 49621 64882, Fax: +7 4962128933

***e-mail nikita.medvedev@desy.de

The presented Monte Carlo model simulates excitation of the electron subsystem of semiconductors by a penetrating swift heavy ion (SHI). The cross sections of interaction of an ion with the electron subsystem of a target are calculated via the complex dielectric function formalism, which accounts for all the collective modes of the electron ensemble of the target. The predicted electron inelastic mean free paths are in a very good agreement with those from the NIST database. The calculated SHI energy losses coincide well with SRIM and CasP codes. The model is used to calculate the spectra of electrons emitted from germanium and silicon targets during SHI irradiation. These spectra agree well with the experimental data.
1 Introduction A swift heavy ion (SHI, $>1 \mathrm{MeV}$ / nucl) losses the largest part of its energy ( $>95 \%$, up to $40 \mathrm{keV} / \mathrm{nm}$ ) for excitation of the electron subsystem of a solid target [1-3]. Fast relaxation of the excess energy of electrons can induce strong material excitation in the nanometric vicinity of the ion trajectory (SHI track) on the femto- to pico-second timescale and can stimulate unusual structure and phase transformations. Such structure modifications of a solid manifest themselves as a latent track in a bulk along the SHI trajectory, and as a surface damage in a shape of craters [4] and/or hillocks [1, 5, 6]. These effects can considerably change target properties, making SHI irradiation a powerful tool for material modifications on the micro- to nano-metric level. Extreme conditions result in unusual kinetic pathways of material excitation and relaxation during SHI irradiations stimulating fundamental interest to this problem.

All the processes in the nanometric vicinity of the SHI trajectory start from excitation of the electron subsystem of a target. Excited electrons spread from the projectile trajectory bringing out the deposited energy. The kinetics of further energy transformations and excitation of the target depend on evolution of the spectra of excited electrons.

Some fraction of fast electrons can be emitted from the sample surface. Detailed information about these electrons gives abilities to verify models developed for description of excitation of the electron ensemble of a target in the nanometric proximity of the SHI trajectory. This information can also be used for elementary analysis of a solid or for design of new types of ion detectors [7].

In this paper, we apply the complex dielectric function (CDF) formalism [8-10] to obtain the cross sections of interaction of a charged particle with matter. The CDF formalism takes automatically into account effects of collective response of the electronic and ionic subsystems of a target to excitations caused by a penetrating particle. The calculated cross sections of SHI and electrons scatterings are then incorporated into the Monte Carlo model to simulate ionization of a target by an incident ion as well as secondary electron cascades produced by fast electrons and Auger decays of holes in deep atomic shells.

The initial version of this MC model has already been successfully applied to quantitative description of the electron kinetics in SHI tracks in some insulators [11-15]. In this work, we extend this model to semiconductors ( $\mathrm{Si}$ and $\mathrm{Ge})$. Comparison of the obtained spectra of emitted electrons with the results of experiments investigating secondary electron emission during irradiation of these materials 
confirms applicability of the developed model to description of the transient electron kinetics in SHI tracks.

2 Monte Carlo model The presented MC model is based on the asymptotic trajectory method of event-by-event simulations of propagation of individual particles [16-20]. The CDF formalism [9] is used to determine the cross sections of an ion and an electron scattering on a target.

In the first Born approximation, the cross-section of interaction of a charged projectile with a spatially and dynamically correlated scattering centers can be represented as a product of the cross section of scattering on an individual scattering center and the "charge-charge" dynamic structure factor [8] of the system of scattering centers. According to the fluctuation-dissipation theorem, this dynamic structure factor of a solid can be expressed in terms of the inverse imaginary part of $\operatorname{CDF} \varepsilon(\omega, q)[9,10,20]$ resulting in the following form of the double differential cross section over the transferred energy $\hbar \omega$ and momentum $\hbar q$ :

$$
\frac{\mathrm{d}^{2} \sigma}{\mathrm{d}(\hbar \omega) \mathrm{d} q}=\frac{2\left(Z_{e} e\right)^{2}}{\pi \hbar^{2} v^{2} n} \frac{1}{q} \operatorname{Im}\left(\frac{-1}{\varepsilon(\omega, q)}\right)
$$

Here, $Z_{e}$ is the effective charge of the projectile penetrating through the scattering system (for an incident electron $Z_{e}=1$, for a heavy ion we apply the Barkas formula [21] to determine $Z_{e}$ ); for inelastic scattering $n=n_{e}$, the density of electrons, or $n=n_{a}$, the density of atoms for elastic scattering; $v$ is the velocity of the incident particle; $e$ is the electron charge; $\hbar$ is the Planck constant. Assuming uniform and homogeneous distribution of scattering centers, the mean free path of a projectile is determined as $\lambda=(n \sigma)^{-1}$, individually for elastic and inelastic scattering cross sections.

The first moment of the cross section (or of the mean free path) gives the energy loss of a projectile:

$$
-\frac{\mathrm{d} E}{\mathrm{~d} x}=\int_{E_{\min }}^{E_{\max }} \frac{\mathrm{d} \lambda^{-1}}{\mathrm{~d}(\hbar \omega)} \hbar \omega \cdot \mathrm{d}(\hbar \omega)
$$

Here $E_{\min }=E_{g}[10,22]$, where $E_{g}$ is the band gap. $E_{g}=1.12 \mathrm{eV}$ for Si [23] and $E_{g}=0.805 \mathrm{eV}$ for Ge [23]. The upper limit for an ion is $E_{\max }=\hbar \omega_{\max }=4 E_{\mathrm{in}} m_{e} M_{\mathrm{in}} /\left(M_{\mathrm{in}}+\right.$ $\left.m_{e}\right)^{2}$, where $E_{\text {in }}$ is the energy and $M_{\text {in }}$ is the mass of an SHI, $m_{e}$ is the free electron mass. For the case of incident electron, $E_{\max }=E_{\text {in }} / 2$ taking into account the identity of electrons.

The integration limits of the double differential inverse mean free path are as follows [10]:

$$
\begin{aligned}
& \frac{\mathrm{d} \lambda^{-1}}{\mathrm{~d}(\hbar \omega)}=\int_{q_{-}}^{q_{+}} \frac{\mathrm{d}^{2} \lambda^{-1}}{\mathrm{~d}(\hbar \omega) \mathrm{d}(\hbar q)} \mathrm{d}(\hbar q), \\
& q_{ \pm}=\sqrt{2 m_{e} / \hbar^{2}}\left(\sqrt{E_{\mathrm{in}}} \pm \sqrt{E_{\mathrm{in}}-\hbar \omega}\right), \text { for electrons; } \\
& q_{-}=\omega / v, \quad q_{+}=\sqrt{2 m_{e} E_{\max }} / \hbar, \text { for ions. }
\end{aligned}
$$

The inverse imaginary part of CDF is reconstructed from the experimentally measured optical coefficients in the form of a set of artificial oscillators [9]:

$$
\operatorname{Im}\left[\frac{-1}{\varepsilon(\omega, q)}\right]=\sum_{i=1}^{n^{\mathrm{os}}} \frac{A_{i} \gamma_{i} \hbar \omega}{\left[\hbar^{2} \omega^{2}-\left(E_{0 i}+\hbar^{2} q^{2} /\left(2 m_{e}\right)\right)^{2}\right]^{2}+\left(\gamma_{i} \hbar \omega\right)^{2}}
$$

where the summation is running through all the oscillators $n_{\mathrm{os}}$; coefficients $E_{0 i}, A_{i}$, and $\gamma_{i}$ for $\mathrm{Si}$ and $\mathrm{Ge}$ are shown in Tables 1 and 2 . These coefficients are carefully chosen to reproduce the low energy $(<50 \mathrm{eV})$ optical constants for most common materials available from Ref. [24] and the data for higher photon energies found in [25]. The algorithm of finding of these coefficients is thoroughly described in Refs. [9, 10, 22].

Tables 1 and 2 also contain the results of the fulfillment of the sum-rules:

$$
Z_{\mathrm{eff}}=2 /\left(\pi \Omega_{p}^{2}\right) \int_{0}^{\omega_{\max }} \operatorname{Im}\left[\varepsilon(\omega, q=0)^{-1}\right] \omega \mathrm{d} \omega
$$

Table 1 The coefficients of the complex dielectric function of silicon in the form of oscillator functions, Eq. (4). The total $p s$-sum gives 0.9897 (1\% deviation from 1).

\begin{tabular}{lllll}
\hline name & $E_{0 i}$ & $A_{i}$ & $\gamma_{i}$ & $f$-sum $\left(N_{e}\right)$ \\
\hline valence band & 16.8 & 278 & 4.1 & $4.1475(4)$ \\
L-shell & 100 & 685 & 145 & $8.0054(8)$ \\
K-shell & 1980 & 168 & 1350 & $1.908(2)$ \\
phonon peak & 0.005 & $1.2 \times 10^{-9}$ & 0.005 & - \\
& 0.02 & $1.5 \times 10^{-8}$ & 0.1 & \\
& 0.076 & $2 \times 10^{-8}$ & 0.005 & \\
total & 0.1 & $5.7 \times 10^{-8}$ & 0.05 & $14.061(14)$ \\
& & & &
\end{tabular}

Table 2 The coefficients of the complex dielectric function of germanium in the form of oscillator functions, Eq. (4). The total ps-sum gives 0.89 (11\% deviation from 1$)$.

\begin{tabular}{lllll}
\hline name & $E_{0 i}$ & \multicolumn{1}{c}{$A_{i}$} & \multicolumn{1}{c}{$\gamma_{i}$} & $f$-sum $\left(N_{e}\right)$ \\
\hline valence band & 17 & 245 & 15 & $4.02(4)$ \\
M-shell Ge & 33 & 1 & 5 & $18.66(18)$ \\
& 75 & 280 & 80 & \\
& 130 & 110 & 80 & \\
& 190 & 750 & 190 & \\
L-shell Ge & 1240 & 675 & 950 & $7.95(8)$ \\
K-shell Ge & 11,210 & 170 & 5500 & $1.86(2)$ \\
phonon peak & 0.012 & $4 \times 10^{-9}$ & 0.005 & - \\
& 0.05 & $1 \times 10^{-7}$ & 0.08 & \\
total & & & & $32.5(32)$ \\
\hline
\end{tabular}


which must be equal to the total number of electrons per molecule of a target, $N_{e}$, when $\hbar \omega_{\max } \rightarrow \infty$. $\Omega_{p}^{2}=4 \pi n_{m} e^{2} / m_{e}, n_{m}$ is the density of molecules of a solid under consideration (so-called $f$-sum rule, or oscillator strength). Another sum-rule, the ps-sum rule or a limiting form of the Kramers-Kronig integral, says that the value

$$
P_{\text {eff }}=2 / \pi \int_{0}^{\hbar \omega_{\max }} \operatorname{Im}\left[\varepsilon(\omega, q=0)^{-1}\right] \mathrm{d}(\hbar \omega) / \hbar \omega
$$

must tend to unity when $\hbar \omega_{\max } \rightarrow \infty$.

The described above CDF formalism gives an ability to calculate the partial cross sections of an electron scattering on the electronic and the atomic subsystems of a solid. It automatically takes into account all collective effects of target electrons, e.g., plasmons, and optical part of lattice collective modes for electron scattering. However, CDF method cannot describe elastic interactions of an SHI due to inapplicability of the first Born approximation to ion-ion collisions.

2.1 SHI passage The MC code simulates penetration of an SHI which incidents perpendicularly to the surface of a layer of $\sim 200 \mathrm{~nm}$ thickness. An ion performs initial ionization of a target creating the first generation of free electrons. Due to heavy mass of a projectile $\left(M_{\mathrm{ion}} \gg m_{e}\right)$ and its negligible elastic scattering on target atoms for energies considered here, the SHI trajectory is assumed to be a straight line.

During penetration of an SHI within a solid, the target electrons are considered as uniformly distributed particles occupying either the atomic energy levels [26] or the states in the valence band according to the density of states (DOS) of the materials ( $\mathrm{Si}$ [27], Ge [28]). Taking into account large velocities of projectiles, we assume these electrons as pointlike particles at fixed positions during their energy and momentum exchange with an SHI.

Spatial propagation of an SHI and its interactions with the electron subsystem of a target are simulated using the Poisson distribution for the free-flight distance [17, 18] with the mean free path of a projectile scattering on a target electrons calculated from the CDF-based cross sections as explained above. Interaction of a projectile with the valence band leads to excitation of plasmons with characteristic energies around of $15-30 \mathrm{eV}$. It is assumed that such plasmon decays instantly into an electron-hole pair [10, 18, 29].

Thus, the transferred energy $\Delta E_{e}$ (and the corresponding zenith scattering angle $\theta$ ) of an ionized electron is calculated from the differential cross section, Eqs. (1)-(4), in accordance with the energy and momentum conservations. The azimuth angle of scattering $\varphi$ is chosen randomly between 0 and $2 \pi$.

The new electron produced in an ionization event by an SHI receives the kinetic energy equal to the difference between $\Delta E_{e}$ and the energy level from which this electron is being ionized. The atomic energy levels are taken from Ref. [30]. A particular energy state in case of a valence band ionization is chosen randomly in accordance with the DOS of the target material.

2.2 Kinetics of electrons Propagation and scatterings of fast electrons are simulated in the same manner as for a swift heavy ion. The kinetic energy of the main part of electrons is significantly larger than the potential energies of their interaction, except for the fraction of the very-lowenergy electrons, which cannot perform new ionizations of atomic shells. Therefore, we can apply the first Born approximation for description of electrons which are able to produce ionization of a target that is in agreement with the CDF formalism describing scattering of such electrons (see above).

We assume that a scattering event of an incident electron on target electrons can result in appearance of only one new electron because multiple electron excitation by a single impact is much less probable [31]. The energy transferred in the collision is determined from the differential cross sections given by Eq. (1).

Because of the limited energies, neither ions nor the produced electrons manifest relativistic effects, Cherenkov, or Bremsstrahlung emissions. All the secondary electrons appearing in cascades are modelled in the same scheme as the primary electrons.

2.3 Auger-decays of deep holes Fast ions as well as high-energy electrons can ionize deep atomic shells. In such collisions, a hole in deep atomic shell is left behind. These holes can relax fast via Auger decays characterized by times typically on femtosecond timescales. For $\mathrm{Si}, K$-shell holes with the ionization potential of $I_{p}(K, \mathrm{Si})=1860 \mathrm{eV}$ have a characteristic Auger-decay of $1.66 \mathrm{fs}$; $L$-shell with $I_{p}(L, \mathrm{Si})=102 \mathrm{eV}$ has the Auger-decay time of $13.3 \mathrm{fs}$ [32]. For Ge, $K$-shell $\left(I_{p}(K, \mathrm{Ge})=11,210 \mathrm{eV}\right)$ decays with the characteristic time of $0.74 \mathrm{fs} ; L$-shell $\left(I_{p}(L, \mathrm{Ge})=1240 \mathrm{eV}\right)$ has the Auger-decay time of $0.91 \mathrm{fs}$; and $M$-shell with the ionization potential of $I_{p}(M, \mathrm{Ge})=33 \mathrm{eV}$ decays with the time constant of $19.6 \mathrm{fs}$ [32].

Auger cascades result eventually in popping up of deepshell holes into the valence band. No radiative decays of deep holes are incorporated into the model owing to domination of Auger processes for light elements [32]. The realized Auger-decay times of holes are determined by the Poisson distribution [11]. The shells, participating in the Auger decay, are chosen randomly. Emission of the Auger electron is assumed uniformly into the solid angle. Emitted electron posses an energy equal to the difference between its own ionization potential and the energy released by a hole jumping up between the energy levels.

2.4 Emission of electrons Emission of an electron from the surface is modelled using potential surface barrier of the Eckart-type [33]. The barrier form depends on three parameters: the material work function $A$, the length $L$ and 
the height $W_{1}$ of the barrier:

$$
\begin{aligned}
& V(z)=-\frac{A \xi}{1-\xi}-\frac{B \xi}{(1-\xi)^{2}}, \\
& \xi=-\exp \left(\frac{2 \pi z}{L}\right) \\
& B=2 W_{1}-A+2 \sqrt{W_{1}^{2}-W_{1} A} .
\end{aligned}
$$

Here, $z$ is the distance from the solid-vacuum interface in the direction perpendicular to the surface.

Work function for $\mathrm{Ge}$ is $4.76 \mathrm{eV}$, and $4.6 \mathrm{eV}$ for $\mathrm{Si}$. The parameters $L$ and $W_{1}$ for Ge are taken from [33], and are assumed to be the same for Si. Ref. [33] proposes the following approximation of the transmission coefficient:

$$
T=\frac{1}{1+\exp \left(\gamma\left(E_{1}-E\right)\right)},
$$

where $E_{1}$ corresponds to the barrier height $W_{1}$. The value of $\gamma$ relates to the characteristic length of the barrier $L$ :

$E_{1}=W_{1}+2 \sqrt{W_{1}\left(W_{1}-A\right)} \cdot\left(\frac{\cosh ^{-1} b}{\delta\left(\sqrt{W_{1}}+\sqrt{W_{1}-A}\right)}-1\right)$, $\gamma=\frac{\gamma_{1} \sinh \left(\gamma_{1}\right)+2 \gamma_{2} \sinh \left(\gamma_{2}\right)}{\sqrt{E_{1}\left(E_{1}-A\right) \cdot\left(b+\cosh \left(\gamma_{1}\right)\right)}}$,

where

$$
\begin{aligned}
& \gamma_{1}=\delta\left(\sqrt{E_{1}}+\sqrt{E_{1}-A}\right), \quad \gamma_{2}=\delta\left(\sqrt{E_{1}}-\sqrt{E_{1}-A}\right), \\
& \delta=\frac{2 \pi L}{h} \sqrt{2 m_{e}}, \quad b=\cosh \left(\pi \sqrt{\frac{8 m_{e} L^{2} B}{h^{2}}-1}\right) .
\end{aligned}
$$

Figure 1 illustrates the dependence of the potential barrier on the distance from the crystal surface for germanium, as well as the dependence of the transmission coefficient on the electron energy obtained from Eqs. (5)-(7).

This method of calculation of the electron transmission coefficient is incorporated into the Monte Carlo code, taking into account the possibilities of reflection and tunneling at the surface potential barrier: when an electron reaches the near-surface region, the corresponding transmission coefficient is calculated using Eq. (6). The energy of the transmitted electron is counted out from the vacuum energy level (the electron kinetic energy minus the work function). Emitted electrons are excluded from further simulation. Non-emitted electrons are reflected from the barrier with the reflection angle equal to the incident angle.

3 Results and discussion Figures Figs. 2 and 3 presents the calculated ion energy losses and the mean free path of electrons in $\mathrm{Ge}$ and $\mathrm{Si}$, using the fitted coefficients of the CDF (Tables 1 and 2) for the numerical integration of

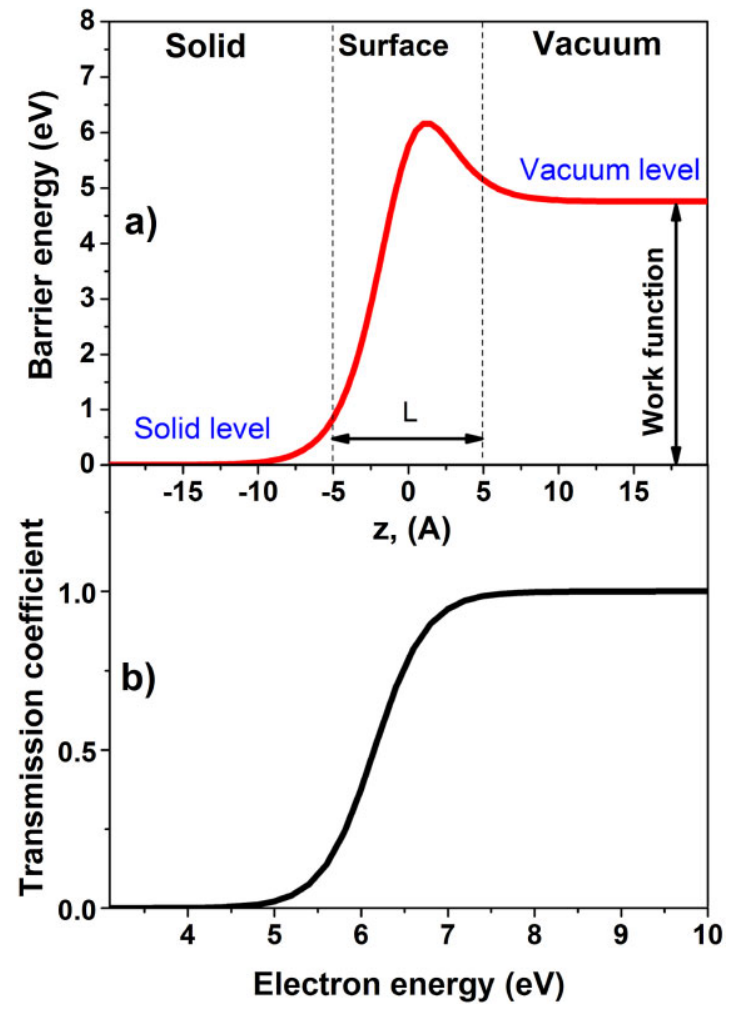

Figure 1 A potential barrier of the Eckart type (a) and the corresponding transmission coefficient (b).

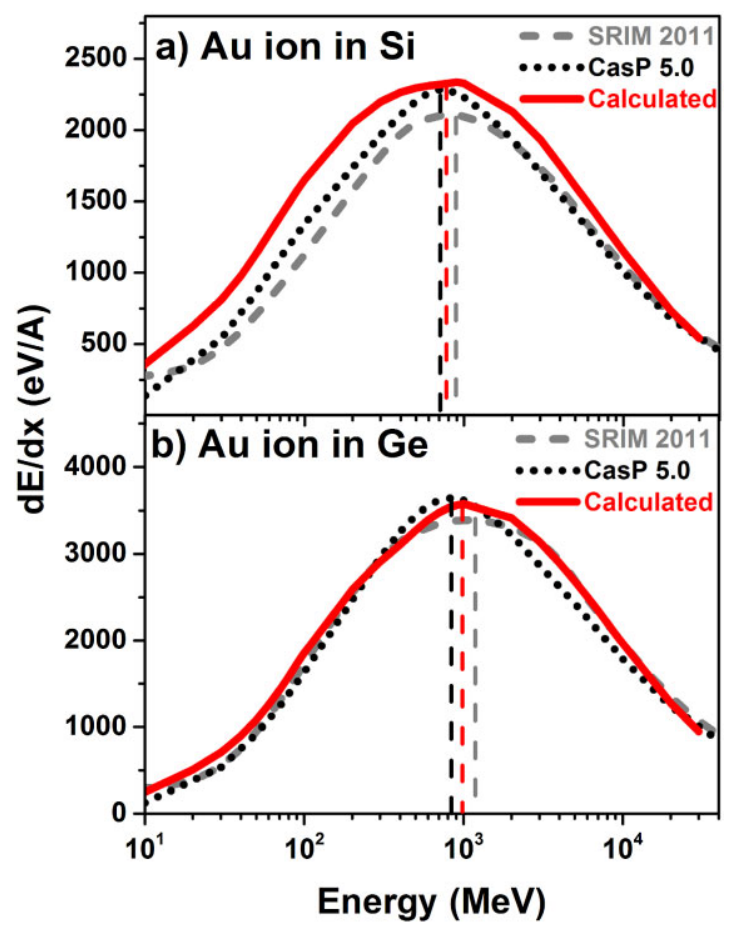

Figure 2 The calculated energy losses of $\mathrm{Au}$ ion in $\mathrm{Si}$ (a) and in $\mathrm{Ge}$ (b) vs. ion energy. 


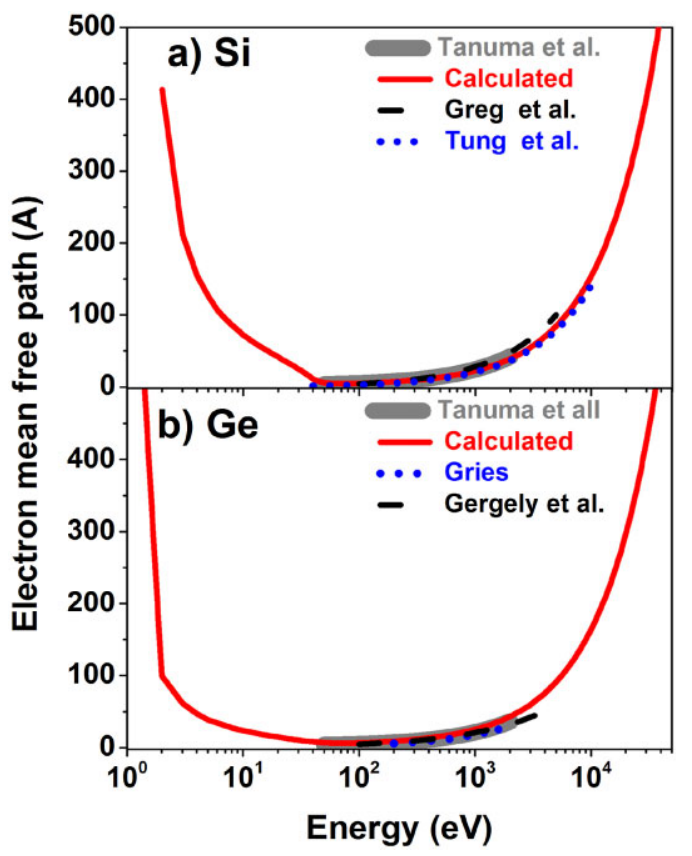

Figure 3 The calculated electron inelastic mean free paths in solid (a) Si and (b) Ge targets.

Eqs. (1)-(4). The results of the calculations with the widely used codes SRIM [34] and CasP [35] for ions, as well as the data from the NIST database for electrons [36] are presented in these figures for comparison. A very good agreement between the presented data is demonstrated with only little deviations due to peculiarities of the compared models. This confirms validity of the calculated cross sections to description of SHI impact and the subsequent kinetics of the electron subsystem of solid $\mathrm{Si}$ and Ge.

The shape of the energy distribution of electrons emitted during $100 \mathrm{fs}$ after passage of Ti $555 \mathrm{MeV}(\mathrm{d} E / \mathrm{d} x=500 \mathrm{eV} / \mathrm{A})$ and $\mathrm{Au} 2187 \mathrm{MeV}(\mathrm{d} E / \mathrm{d} x=3200 \mathrm{eV} / \mathrm{A})$ ions in $\mathrm{Ge}$ are compared in Fig. 4 with the experimental data of secondary electron emission stimulated by electron bombardment [37]. The curves are normalized to the integrated value.

It should be noted that such a comparison is possible because the emission spectrum consists mainly of secondary electrons produced by fast primary $\delta$-electrons in ionization cascades. The majority of primary $\delta$-electrons is moving in the parallel direction to the solid surface (perpendicular to the SHI trajectory). Therefore, the starting time of electron emission is related to the time of randomization of the ionized electrons directions (a few tens of fs). One can see from Fig. 4 that the low energy part of primary spectrum is similar for SHIs of different masses and energies (the Ti and $\mathrm{Au}$ ions induced emission spectra).

Figure $4 \mathrm{a}$ demonstrates good coincidence with experimental data for Ge. The left, increasing part of the spectrum depends considerably on the surface barrier form, while the cross sections of interaction of an electron with a solid are responsible for the slope of the decreasing right part. In

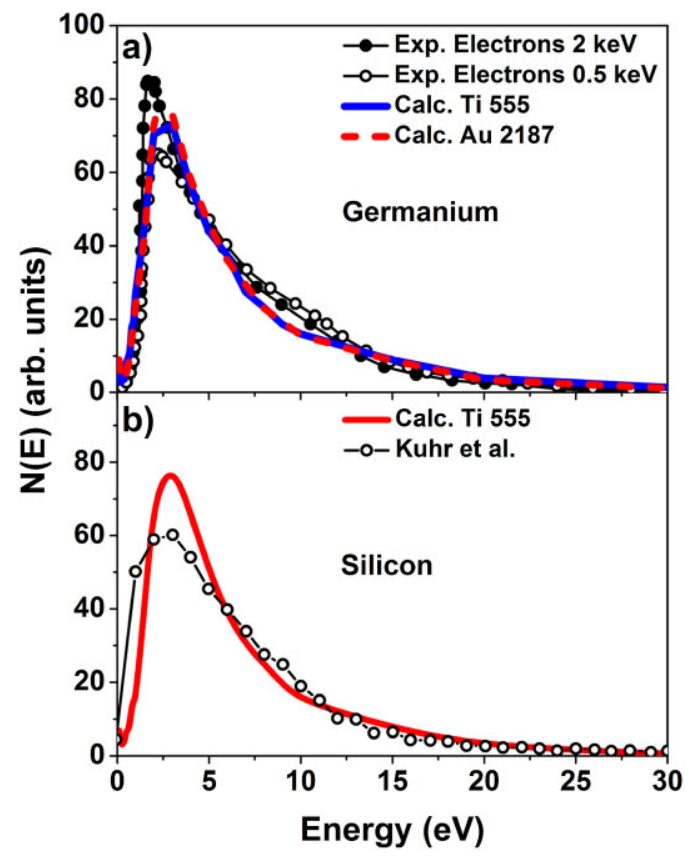

Figure 4 Energy distributions of the secondary emitted electrons in (a) Ge compared with experimental data [37], (b) Si compared with MC calculations from [20].

Fig. $4 \mathrm{~b}$ this typical shape of the emitted electrons spectra appears also for the case of $\mathrm{Si}$ coinciding reasonably well with other Monte Carlo simulations from Ref. [20].

The spectrum of the emitted electrons from $\mathrm{Si}$ irradiated with Ti-ion $(555 \mathrm{MeV})$ is shown in Fig. 5 for the energy range up to $10 \mathrm{keV}$. It is seen that there are a few characteristic features of the spectrum: decays of plasmons create a slight "shoulder" at the energies of $\sim 15 \mathrm{eV}$. A peak formed by Auger-electrons emitted after L-shell hole decay appears at the energies of 70-80 eV. K-shell Auger decays produce an additional minor peak at the energies of 1.6$1.7 \mathrm{keV}$. These features of the emitted electron spectra could

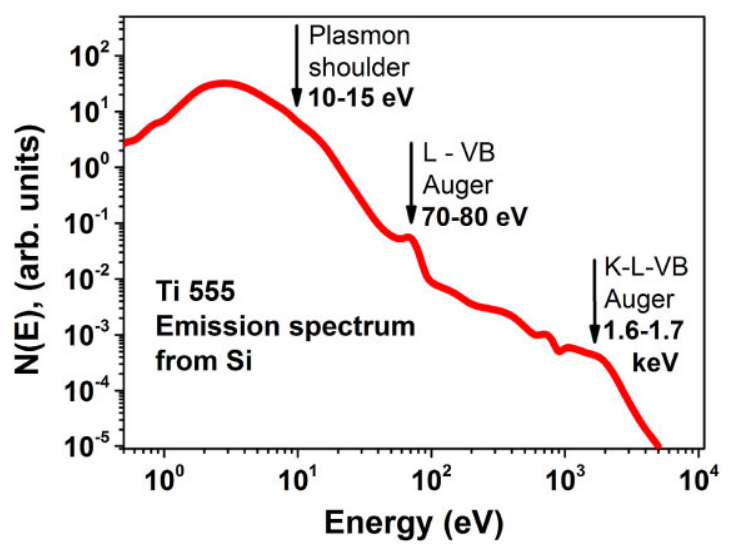

Figure 5 The spectrum of the emitted electrons in $\mathrm{Si}$ after $\mathrm{Ti}$ ion impact (555 MeV energy). 
be used for testing of different models and against experimental data.

4 Conclusions The presented Monte Carlo (MC) model is based on the CDF formalism taking into account collective response of a target to excitations induced by a penetrating charged particle. This allows determining with high accuracy the cross sections of an ion and an electron inelastic scattering in a solid $\mathrm{Si}$ and Ge. The presented parameterized CDF coefficients provide very good agreement of the ion energy losses with SRIM [34] and CasP [35] codes as well as the calculated electron mean free paths with those from the NIST database [36].

The reliability of the obtained cross section is also confirmed by a good coincidence of the Monte Carlo modelled spectra of emitted electrons with those observed in experiments. This verifies good applicability of the developed model for calculations of the transient electron kinetics in SHI tracks.

Acknowledgements A. E. Volkov acknowledges Grant 1302-1020 ofi-m from Russian Foundation for Basic Research.

\section{References}

[1] F. Aumayr, S. Facsko, A. S. El-Said, C. Trautmann, and M. Schleberger, J. Phys.: Condens. Matter 23, 393001 (2011).

[2] A. M. Stoneham and N. Itoh, Appl. Surf. Sci. 168, 186 (2000).

[3] F. F. Komarov, Physics - Uspekhi 46, 1253 (2003).

[4] D. Fink (ed.), Fundamentals of Ion-Irradiated Polymers (Springer, Berlin Heidelberg, Berlin, 2004), p. 406.

[5] N. A. Medvedev, K. Schwartz, C. Trautmann, and A. E. Volkov, Phys. Status Solidi B 250, 850 (2013).

[6] K. Schwartz, A. Volkov, M. Sorokin, C. Trautmann, K.-O. Voss, R. Neumann, and M. Lang, Phys. Rev. B 78, 024120 (2008).

[7] J. C. Faivre, H. Fanet, A. Garin, J. P. Robert, M. Rouger, and J. Saudinos, IEEE Trans. Nucl. Sci. 24, 299 (1977).

[8] L. Van Hove, Phys. Rev. 95, 249 (1954).

[9] R. H. Ritchie and A. Howie, Philos. Mag. 36, 463 (1977).

[10] A. Akkerman, T. Boutboul, A. Breskin, R. Chechik, A. Gibrekhterman, and Y. Lifshitz, Phys. Status Solidi B 198, 769 (1996)

[11] N. A. Medvedev, A. E. Volkov, N. S. Shcheblanov, and B. Rethfeld, Phys. Rev. B 82, 125425 (2010).

[12] N. A. Medvedev, A. E. Volkov, K. Schwartz, and C. Trautmann, Phys. Rev. B 87, 104103 (2013).
[13] N. A. Medvedev, R. A. Rymzhanov, and A. E. Volkov, Nucl. Instrum. Methods Phys. Res. B 315, 85 (2013).

[14] R. A. Rymzhanov, N. A. Medvedev, and A. E. Volkov, Nucl. Instrum. Methods Phys. Res. B 326, 238 (2014).

[15] S. A. Gorbunov, N. A. Medvedev, R. A. Rymzhanov, P. N. Terekhin, and A. E. Volkov, Nucl. Instrum. Methods Phys. Res. B 236, 163-168 (2014).

[16] W. Eckstein, Computer Simulation of Ion-Solid Interactions (Springer, Berlin, Heidelberg, 1991).

[17] B. Gervais and S. Bouffard, Nucl. Instrum. Methods Phys. Res. B 88, 355 (1994).

[18] A. Akkerman, M. Murat, and J. Barak, Nucl. Instrum. Methods Phys. Res. B 269, 1630 (2011).

[19] C. Jacoboni and L. Reggiani, Rev. Mod. Phys. 55, 645 (1983).

[20] J.-C. Kuhr and H.-J. Fitting, J. Electron Spectrosc. Relat. Phenom. 105, 257 (1999).

[21] H.-D. Betz, Rev. Mod. Phys. 44, 465 (1972).

[22] N. Medvedev, AIP Conf. Proc. 582, 582 (2012).

[23] W. Strehlow and E. Cook, Phys. Chem. Ref. Data 2, 163 (1973).

[24] E. D. Palik, Handbook of Optical Constants of Solids (Academic Press, San Diego, 1985), p. 804.

[25] B. L. Henke, E. M. Gullikson, and J. C. Davis, At. Data Nucl. Data Tables 54, 181 (1993).

[26] A. A. Baranov, N. A. Medvedev, A. E. Volkov, and N. S. Scheblanov, Nucl. Instrum. Methods Phys. Res. B 286, 51 (2012).

[27] E. O. Kane, Phys. Rev. 146, 558 (1966).

[28] M. Thorpe and D. Weaire, in: Amorphous and Liquid Semiconductors, edited by J. Stuke and W. Brenig (Taylor and Francis, London, 1974).

[29] A. Akkerman, J. Barak, and D. Emfietzoglou, Nucl. Instrum. Methods Phys. Res. B 227, 319 (2005).

[30] J. A. Bearden and A. F. Burr, Rev. Mod. Phys. 39, 125 (1967).

[31] B. Gervais, M. Beuve, G. H. Olivera, and M. E. Galassi, Radiat. Phys. Chem. 75, 493 (2006).

[32] O. Keski-Rahkonen and M. O. Krause, At. Data Nucl. Data Tables 14, 139 (1974).

[33] C. Bouchard and J. D. Carette, Surf. Sci. 100, 251 (1980).

[34] J. P. Ziegler, U. Biersack, and J. F. Littmark, The Stopping and Range of Ions in Solids (Pergamon Press, New York, 1985).

[35] P. P. L. Grande and G. Schiwietz, Nucl. Instrum. Methods Phys. Res. B 267, 859 (2009).

[36] C. J. Powell and A. Jablonsky, NIST Electron Inelastic-MeanFree-Path Database, www.nist.gov/srd/nist71.cfm.

[37] P. W. Palmberg, J. Appl. Phys. 38, 2137 (1967). 